\title{
Emotion-Induced Amnesia in Rats: Working Memory-Specific Impairment, Corticosterone-Memory Correlation, and Fear Versus Arousal Effects on Memory
}

\author{
James C. Woodson, ${ }^{1,3}$ Deric Macintosh, ${ }^{4}$ Monika Fleshner, ${ }^{4}$ and David M. Diamond ${ }^{1,2,3,5}$ \\ ${ }^{1}$ Departments of Psychology and Pharmacology, ${ }^{2}$ University of South Florida, Tampa, Florida 33620, USA; ${ }^{3}$ Division of Medical \\ Research, VA Medical Center, Tampa, Florida 33612, USA; ${ }^{4}$ Department of Kinesiology and Applied Physiology, and Center \\ for Neuroscience, University of Colorado, Boulder, Colorado, USA
}

\begin{abstract}
We have shown previously that psychological stress (predator exposure) impairs spatial memory in rats. We have extended that finding here to show that predator stress selectively impaired recently acquired (hippocampaldependent) spatial working memory without affecting long-term (hippocampal-independent) spatial reference memory. We also investigated why predator exposure impairs memory. Was spatial memory impaired because of the fear-provoking aspects of predator exposure or only because the cat was a novel and arousing stimulus? If the latter possibility was correct, then any novel and arousing stimulus, independent of its emotional valence (i.e., aversive or appetitive), would impair memory. We found that spatial working memory was not impaired when the male rats were exposed to a sexually receptive female rat, a stimulus that was novel and arousing to them, but not aversive. We also found that there was an equivalent increase in serum corticosterone levels in male rats exposed to either a cat or a female rat, but only the cat-exposed rats exhibited a significant correlation between corticosterone levels and impaired memory. Overall, this series of experiments demonstrates that (1) predator stress selectively impaired working (hippocampal-dependent), but not reference (hippocampal-independent), memory; (2) a fear-provoking stimulus, and not merely novelty and increased arousal, impaired spatial memory; and (3) increased corticosterone levels correlated with impaired spatial working memory only under predator exposure, that is, fear-provoking conditions.
\end{abstract}

Extensive research has shown that arousing stimuli exert profound and long-lasting influences on memory formation. Much of the literature has focused on the finding that increased arousal enhances memory (Cahill 2000; LeDoux 2000; McGaugh 2000). However, increased emotionality, and especially stress, also impairs memory (Diamond and Park 2000; Kim and Diamond 2002), and can induce amnesia (Loftus and Kaufman 1992; Joseph 1999). Studies on rodents and people indicate that stress or glucocorticoid administration can impair hippocampus-specific learning and memory (Kirschbaum et al. 1996; de Quervain et al. 1998, 2000; Diamond et al. 1999b; Lupien and Lepage 2001; Wolf et al. 2001; Payne et al. 2002). In addition, stress or glucocorticoids block hippocampal long-term (LTP) and primed burst (PBP) potentiation (Foy et al. 1987; Diamond et al. 1994; Akirav and Richter-Levin 1999; Mesches et al. 1999; Pavlides and McEwen 1999; Kim et al. 2001; Alfarez et al. 2002), two well-described physiological models of memory. Thus, strong evidence indicates that stress or glucocorticoids can adversely affect hippocampal processing.

The findings of hippocampal sensitivity to stress are well documented, but our understanding of how emotionality affects hippocampal versus nonhippocampal processing is still in its infancy, and many issues remain unresolved. For example, rats exposed to threatening environments, such as, predator exposure, exhibit impaired performance in spatial memory tasks (Diamond

\section{${ }^{5}$ Corresponding author.}

E-MAIL ddiamond@chuma1.cas.usf.edu; FAX (813) 974-4617.

Article and publication are at http://www.learnmem.org/cgi/doi/10.1101/ Im.62903. et al. 1996, 1999b). One interpretation of these findings is that the stressors produced a memory-specific impairment. However, another interpretation of the findings was that predator exposure produced a change in the rat's behavior that interfered with performance, including changes in motor activity or impaired attention to a task. It was also not known whether cat exposure impaired performance because the cat was threatening to the rat or only because the cat was a novel and arousing stimulus. Perhaps any arousing stimulus, including one that was appetitive, would have produced the same adverse effects on memory as were produced by predator exposure.

Finally, the mechanisms by which stress affects hippocam pal-dependent memory are not well understood. For example, the relationships among glucocorticoids, stress and memory are complex. Elevated glucocorticoid levels are commonly viewed as a sufficient physiological indicator of a stress state, but glucocorticoid levels can also be elevated in response to conditions that are not typically considered to be stressful, such as during feeding, sex, and exercise (Moberg et al. 1975; Phoenix et al. 1977; Bronson and Desjardins 1982; Rosmond et al. 2000; Kanaley et al. 2001; Makatsori et al. 2003). Moreover, glucocorticoids (corticosterone in rats and cortisol in people) have been associated with enhanced (Sandi et al. 1997; Roozendaal 2000; Buchanan and Lovallo 2001) as well as impaired (Kirschbaum et al. 1996; de Quervain et al. 1998, 2000; Mendl 1999; Newcomer et al. 1999) memory. These findings illustrate our incomplete understanding of the complexity of glucocorticoid-memory interactions.

The current experiments addressed these issues with an extensive characterization of the effects of arousing experiences on 
different forms of spatial memory. In one experiment, we compared the effects of predator exposure on spatial working memory, which is dependent on hippocampal functioning (Whishaw 1985; Bohbot et al. 1996; Diamond et al. 1999b; Steele and Morris 1999), versus retrieval of spatial reference memory, which is not dependent of hippocampal functioning (Olton and Papas 1979; Eichenbaum et al. 1990; Kitajima et al. 1992). In another experiment, we compared the effects of appetitive (sexual) versus aversive (fear-provoking) stimuli on spatial working memory, with a concomitant assessment of serum corticosterone and testosterone levels. This series of experiments, therefore, provides a novel perspective on how different emotionally evocative conditions affect different forms of spatial memory.

\section{RESULTS}

\section{Experiment 1}

Two forms of spatial memory are well described in rodent behavioral studies, working and reference memory. Working memory is a dynamic encoding process, whereby information is acquired and updated repeatedly with new information (Olton et al. 1979; Diamond et al. 1996). In working memory testing in the water maze, animals are trained to learn a new hidden platform location on each day of training (Whishaw 1985; Bohbot et al. 1996; Steele and Morris 1999). Spatial working memory is impaired by the disruption of hippocampal functioning (Ohno et al. 1993; Bohbot et al. 1996; Steele and Morris 1999). Reference memory, in contrast, is a form of long-term memory, in which the information to be remembered remains unchanged across many days of training. In reference memory testing in the water maze, the hidden platform is at the same location on every trial for every day of training. After several days to weeks of training, the memory of the platform location becomes consolidated, presumably at extra-hippocampal sites. Following extensive preliminary training, retrieval of reference memory is not impaired by manipulations that disrupt hippocampal activity (Olton et al. 1977, 1979; Eichenbaum et al. 1990; Kitajima et al. 1992).

Experiment 1 evaluated the effects of predator exposure on working and reference memory for two reasons. First, working, but not reference memory was impaired by stress in rats trained to remember the location of food in a radial arm maze (Diamond et al. 1996). Second, working memory was impaired in hippocampal-lesioned and stressed intact rats trained in the radial arm water maze (RAWM; Diamond et al. 1999b). The latter study thereby demonstrated the dependence of the task on the integrity of the hippocampus, as well as its susceptibility to stress. The current study was designed to replicate and extend the previous work by investigating predator stress effects on working versus reference memory in rats trained in the RAWM.

It is also important to note that intense arousal can affect brain and behavioral responses to sensory stimulation (Bindra 1959; Sokolov 1963; Richardson et al. 1988; Lane et al. 1999). Therefore, it was possible that predator exposure effects on memory described in our previous work (Diamond et al. 1999b) may have reflected changes in sensory, motor, or motivational aspects of rat behavior, independent of stress-induced changes in memory-related processing. In Experiment 1, the inclusion of reference memory-trained rats provided a diagnostic of the degree to which predator exposure might affect performance variables, as well as an assessment of the effects of acute stress on well-consolidated spatial memory.

In Experiment 1, working memory-trained rats reached the performance criterion (described in Materials and Methods) in a mean of $12 \mathrm{~d}$ of training. By reaching the performance criterion, all rats in the working memory group attained a high degree of proficiency in their capacity to learn, and then remember, for 30 min, a new location of the hidden platform on each day of training. Reference memory-trained rats were also given $12 \mathrm{~d}$ of watermaze training before the experimental manipulations were conducted. For the reference memory-trained group, the platform was always in the same location on every trial for every day of training. On the two days when predator exposure was introduced, that is, on the $13^{\text {th }}$ and $14^{\text {th }}$ days of training, the reference memory-trained rats already had a well-established memory of the platform location. The working memory group also had $12 \mathrm{~d}$ of preliminary training, but they had learned that the hidden platform was in a different location on each day of training. Thus, reference memory-trained rats had a long-term memory of the platform location that had formed over $12 \mathrm{~d}$ of training, and working memory-trained rats had a newly formed memory of the platform location when the two groups, for the first time, were placed near the cat. If stress produced nonspecific changes in memory, motor activity, or attention to the task, then stressinduced impairments in performance would be evident in both working- and reference-memory trained groups. If, on the other hand, predator stress effects were specific to hippocampal-type processing, then the impairments in performance would be expected to occur only for rats trained in the working memory task.

\section{Findings}

Data were obtained from three groups. Two of the groups were given working memory (WM) training. Rats in one of the WMtrained groups were placed in a familiar environment, their home cage (HC), during the 30-min delay period between the acquisition and memory test phases, that is, between Trial 4 (T4) and the Retention Trial (RT), (WM-HC; $n=7)$. Rats in the other WM-trained group were placed in a chamber with a cat during the 30-min delay period (WM-Cat; $n=9$ ). The third group was given reference memory (RM) training. Rats in this group were placed in the chamber with a cat during the delay period (RMCat; $n=8)$. The two WM-trained groups had a mean of $12 \mathrm{~d}$ of training to reach the performance criterion. The RM-trained rats, therefore, were given an equivalent number of days of preliminary training. The data presented here were obtained on the $2 \mathrm{~d}$ in which the stress or control manipulations took place after the $12 \mathrm{~d}$ of preliminary training was completed.

\section{Acquisition Phase (Trials 1-4)}

The acquisition curves in Figure 1 indicate that all three groups had learned the procedural elements of their respective tasks. The large number of errors committed on Trial 1 (T1) by the two WM-trained groups (WM-Cat and WM-HC) occurred because the rats in these groups searched the maze on the first trial of the day to find the new location of the hidden platform. The two WM groups, therefore, showed within-day learning curves, as indicated by significant decreases in the number of errors across T1T4. In contrast, rats in the RM trained, cat-exposed (RM-Cat) group made very few errors on T1 because they had already learned the constant location of the platform during the previous $12 \mathrm{~d}$ of training.

The three groups exhibited equivalent performance on T3 and T4, which were the last two acquisition trials before the rats were placed either in their home cages (WM-HC) or with a cat (WM-Cat and RM-Cat) during the 30-min delay period. At the end of the delay period, all rats were returned to the RAWM for spatial memory testing. It is important to emphasize that although the three groups were statistically equivalent on T3 and $\mathrm{T} 4$, the performance by the RM-trained group represented longterm memory formed over the 12 previous days of training, and performance by the 2 WM-trained groups represented a newly 


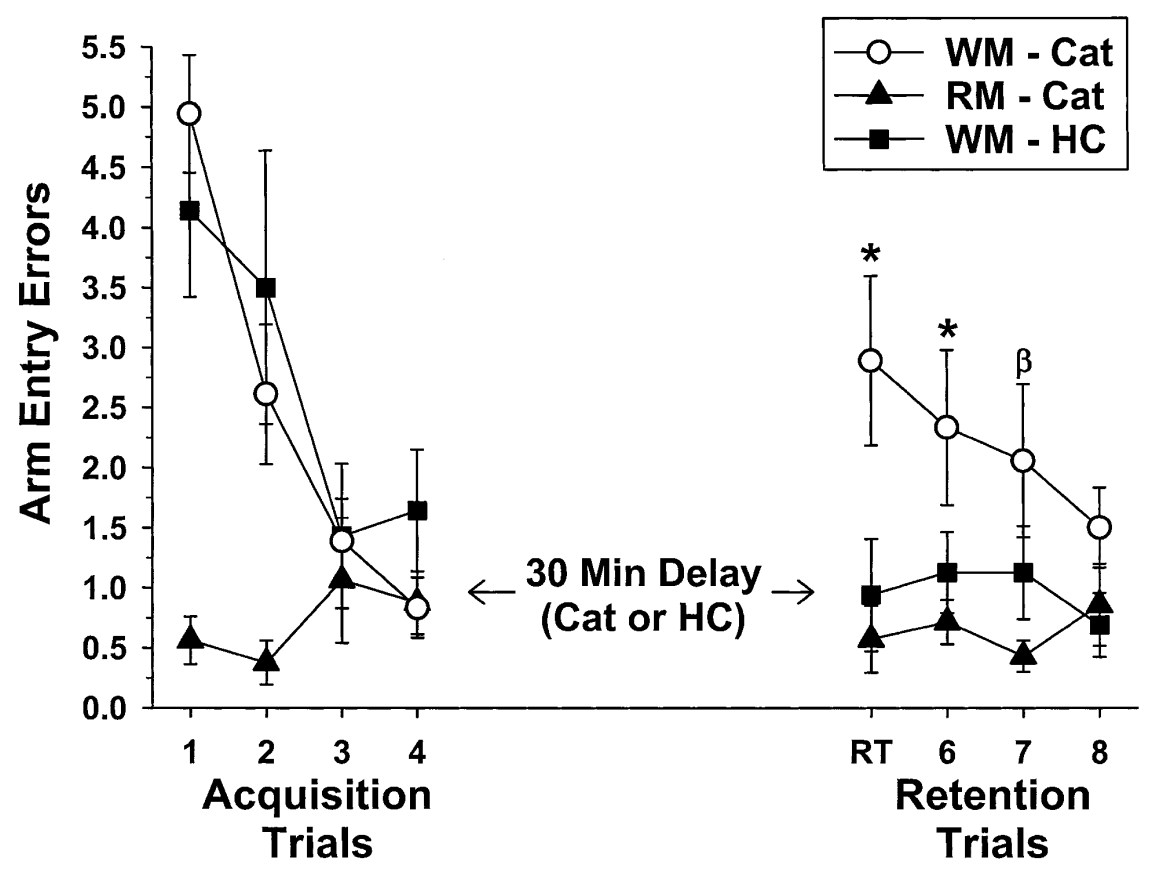

Figure 1 Mean ( \pm SEM) errors for three groups of rats trained in the working-memory (WM) or reference-memory (RM) versions of the radial-arm water maze (RAWM). The WM-Cat (exposure) and RM-Cat (exposure) groups spent the 30-min delay period between the acquisition (T1-T4) and retention (RT-T8) phases in a chamber with a cat and the WM-HC (HC; home cage exposure) group spent the 30-min delay period in their home cages. Here and in Figures 2-4, RT refers to retention trial, emphasizing that at the end of the delay period, the RT measured a rat's retention of the memory for the platform location. The three groups all had a mean of 12 days of training before they were given either the control (home cage) or stress (cat exposure) manipulation. The * indicates that on the RT and T6, the WM-Cat group was significantly different from both the RM-Cat and WM-HC groups, and the $\beta$ indicates that on T7, the WM-Cat group was significantly different from the RM-Cat group.

acquired memory of the platform location formed in $~ 2-3$ min during T1-T4 on that day.

Mixed design ANOVAs (Across Groups $\times$ Within Trials) for the Acquisition Phase revealed a significant effect of Group, $F(2$, $21)=16.48, P<0.01$ and a significant effect of Trial, $F(3$, $63)=10.28, P<0.01$ and a significant Group $\times$ Trial interaction, $F(6,63)=4.73, P<0.01$. Analysis of the interaction revealed that the two WM groups (WM-HC and WM-Cat) did not differ significantly from each other on any acquisition trial. On T1 and T2, the WM groups made significantly more errors than the RMCat group $(P<0.01)$. The three groups' performance did not differ significantly on T3 and T4 $(P>0.05)$.

\section{Retention Phase (Trials 5-8)}

Trial 5 is referred to as the retention trial (RT), because this trial was the critical measure of memory across the 30-min delay period. The WM-HC and RM-Cat groups both exhibited good spatial memory on the RT, which was significantly better than performance on this trial by the WM-Cat group. ANOVA revealed an effect of Group, $F(2,21)=7.91, P<0.01$, with no effect of Trial and no interactions. The WM-HC and RM-Cat groups did not differ significantly from one another on RT-T8. Planned pairwise comparisons indicated that rats in the WM-Cat group made significantly more errors on the RT and T6 than rats in the WM-HC $(P<0.05)$ group and were different from the RM-Cat group on the RT, T6, and T7 $(P<0.05)$.

Thus, cat exposure selectively impaired working, but not reference memory. It is notable that the WM-Cat group was able to relearn the within-day location of the platform, achieving a level of performance that was equivalent to that of the WM-HC group on $\mathrm{T} 7$ and T8. Thus, cat exposure disrupted WM (as indicated by impaired performance on RT and T6), followed by evidence of an intact ability of the cat-exposed group to relearn the location of the platform, as indicated by good performance, equivalent to that of the other two groups, on $\mathrm{T} 7$ and $\mathrm{T} 8$.

\section{Experiment 2}

The findings from Experiment 1 demonstrated that cat exposure did not affect the rat's capacity to perform the procedural elements of the task or in the motivation of the rat to escape from the water. Experiment 1, therefore, showed that working (hippocampal-dependent) memory is more susceptible to impairment by stress than is long-term (hippocampal-independent) memory. Experiment 2 further explored the nature of the stress-induced WM impairment.

One well-studied effect of cat exposure is that it evokes an instinctual fear response in rats (Blanchard et al. 1993; Dielenberg et al. 2001), and it may have been the manifestations of fear, per se, that impaired their memory. It was also possible that cat exposure impaired WM because of the novel and arousing aspects of the cat, independent of its fear-provoking characteristics. It is known that novelty alone can impair spatial memory (Diamond et al. 1996) and can block hippocampal synaptic plasticity (Diamond et al. 1990, 1994; Xu et al. 1997, 1998). Thus, cat exposure may have impaired WM solely, because the cat was a novel and arousing stimulus, independent of its fear-provoking characteristics. If novelty alone were sufficient to impair WM, then a different stimulus, one that is novel and arousing but lacks a fear component, should also impair WM. Experiment 2 tested the possibility that male rats exposed to a potent novel and arousing stimulus, a sexually receptive female rat, during the 30-min delay period between the acquisition and memory test trials, would exhibit the same degree of retrograde amnesia as male rats exposed to a cat. We addressed this aim with two manipulations involving estrous female rats. One group of male rats was allowed physical contact with the female and a second group was exposed to the visual, olfactory, and auditory aspects of the female, but physical contact was not possible. The latter group tested the possibility that frustration, that is, the lack of ability of these males to gain access to the female, would provide a strong distraction that might impair their WM.

Glucocorticoids provide one measure of an animal's level of arousal, because they are the end component of hypothalamicpituitary-adrenal (HPA) axis activation (Miller and O'Callaghan 2002). In addition, testosterone levels are sensitive to both stress and sexual activity (Niikura et al. 2002). Therefore, we obtained two endocrine indicators of stress and arousal by assaying corticosterone and testosterone levels from blood samples obtained from the rats immediately after the last test trial (T8) was completed.

\section{Findings}

Working memory on the RT, corticosterone, and testosterone levels of the groups of rats with versus, without direct female 
contact did not differ significantly [female contact vs. noncontact frustration comparisons, $1.3( \pm 0.3)$ vs. $1.2( \pm 0.5)$ memory (RT) errors; testosterone, $0.12( \pm 0.04)$ vs. $0.09( \pm 0.03) \mu \mathrm{g} / \mathrm{dl}$; corticosterone, 37.0 ( \pm 6.3 ) vs. 24.3 ( \pm 4.5 ), all $P>0.05$, $t$-test]. Thus, there was no frustration versus female contact effect on spatial memory or in the endocrine measures. Therefore, the data for these two groups were combined to form one group, which is referred to as the Female Exposure group $(n=10)$, to increase the power of the statistical analyses. All analyses were then conducted on three groups, which were exposed to the cat $(n=7)$, female rat $(n=10)$, or novel environment $(n=7)$.

All male rats exposed to a female rat were active for the entire 30-min exposure period, with their attention focused primarily in the direction of the female rat. The five male rats restricted from gaining access to the female were continuously active within their enclosure for the entire duration of the delay period. Each of the five males that had access to a female typically spent the first 15-25 min exhibiting continuous motor activity in the vicinity of the female, followed by the majority of the male rats exhibiting stereotypic sexual behaviors, that is, mounting, intromission, and ejaculation.

\section{Acquisition Phase (T1-T4)}

The three groups (cat, female rat, novel environment) showed equivalent acquisition curves on Trials $1-4$, as evidenced by a significant effect of Trial, $F(3,63)=20.24, P<0.001$, with no effect of Group and no Trial $\times$ Group interactions (Fig. 2).

\section{Retention Phase (Trials RT-T8)}

Overall, there was a significant effect of Group, $F(2,21)=14.82$, $P<0.001$, with no effect of Trial and no interactions. The explicit purpose in the design of this study was to evaluate the effects of manipulations occurring during the 30-min delay period on per- formance on the RT. If there were impairments on the RT, then the a priori predictions were that there might also be impairments on subsequent (T6-T8) trials. Planned comparison analysis of RT performance showed that the Novel Environment and Female-Exposure groups performed at an equivalent level, which indicated that these two groups remembered the platform location equally well. The Cat-Exposed group, in contrast, exhibited a working-memory deficit. Their performance differed significantly from the other two groups on the RT $(P>0.05)$. In addition, the Cat-Exposed group's WM performance was significantly worse than that of the Novel environment group on T6-T8 and was worse than that of the Female-Exposed group on T8 (Bonferroni-corrected $t$-tests, $P<0.05$ ).

\section{Fear-Dependent Correlations Between Corticosterone Levels and Memory}

Serum testosterone and corticosterone levels and performance on the memory trial (RT) are presented in Figure 3. Testosterone levels were not altered significantly by any of the test conditions. Corticosterone was significantly elevated in the cat- and femaleexposed rats, in comparison with the group placed in the novel environment. Importantly, although corticosterone levels were elevated to an equivalent extent in the cat- and female rat-exposed rats, only the cat-exposed group committed a significant increase in the number of their WM errors (Fig. 3).

A further assessment of the corticosterone-memory interaction is displayed in Figure 4. A regression analysis was conducted on corticosterone levels and memory performance from control rats and either cat- or female rat-exposed animals. The assumption in this analysis was that there would be a positive corticosterone-memory correlation that would extend from the baseline to the increase in levels produced by either cat or female-rat exposure. With cat- but not female-rat exposure, there was a significant positive linear correlation between corticosterone levels and memory (RT) errors. That is, increased corticosterone correlated with greater memory impairments (more errors on the RT) in the combined baseline + cat-exposed groups (top) $(r=0.68, P<0.05)$, but not in the baseline + female-rat exposed group (bottom) $(r=0.24, P>0.1)$.

\section{DISCUSSION}

It is widely recognized that acute stress can interfere with cognitive and electrophysiological measures of hippocampal functioning (Lupien and McEwen 1997; de Kloet et al. 1999; Kim and Diamond 2002). There are important issues, however, that have not been addressed in substantiating the stress-memory-hippocampus connection. For example, there is evidence that stress, for example, pawshock, an unfamiliar environment, or predator exposure in rats (Diamond et al. 1990, 1994, 1996, 1999b, 2001; Lupien and McEwen 1997; Kim et al. 2001; Kim and Diamond 2002) or public speaking in people (Kirschbaum et al. 1996; Wolf et al. 2001; Payne et al. 2002), can impair hippocampal-dependent memory. However, nonstressful arousing experiences can also affect memory (Christianson 1986; Bradley et al. 1992; Bush and Geer 2001; Newell et al. 2001), suggesting the possibility that any

Figure 2 Mean ( \pm SEM) errors for three groups of rats trained in the working memory version of the RAWM. One group was exposed to a female rat (Female Rat), another was exposed to a cat (Cat), and a third was exposed only to a novel environment (Novel Box) for the first 30 min of the 45-min delay between the Acquisition and Retention Phases of testing. All rats spent the final 15 min of the delay period in their home cages. The * indicates that the cat-exposed group was significantly different on RT and T8 from both Novel and Female-exposed groups, and the $\beta$ indicates that the Cat-exposed group was significantly different from the Novel group on T6 and T7 $(P<0.05)$. 

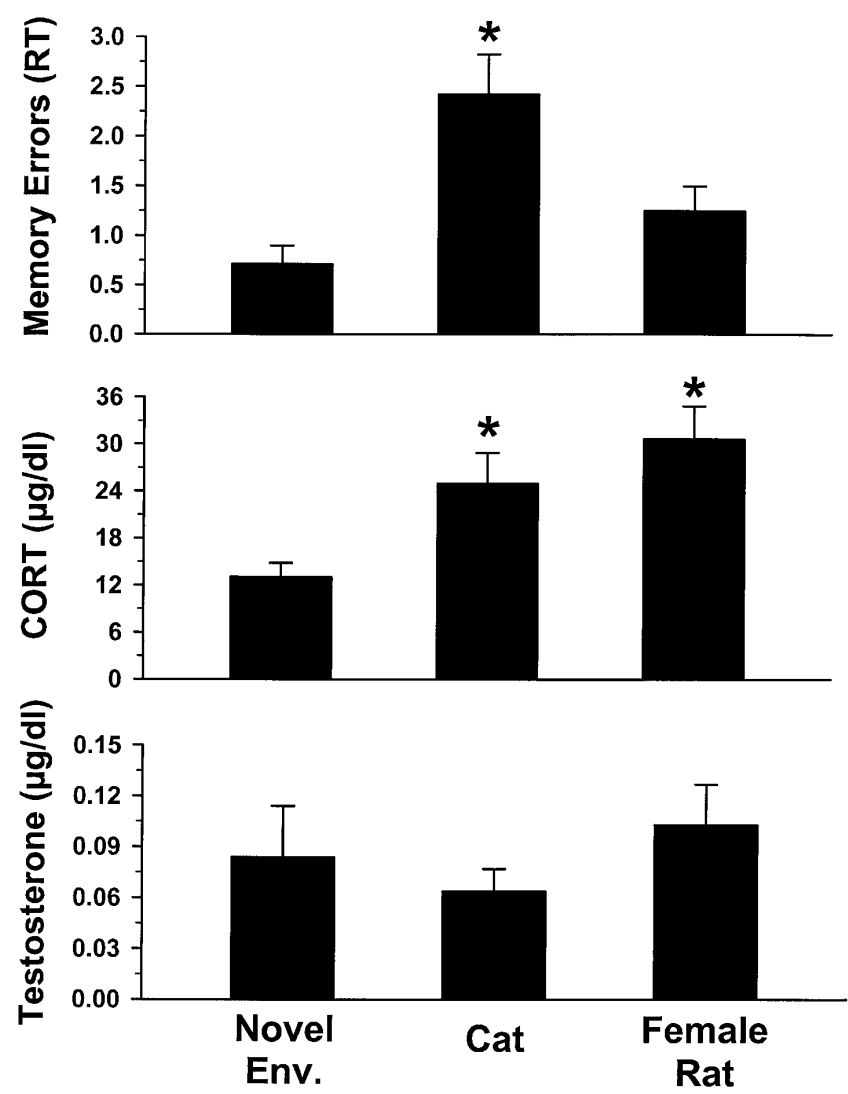

Figure 3 Mean ( \pm SEM) data for memory errors (RT data from Fig. 3), corticosterone and testosterone levels for groups exposed to the novel environment, cat or an estrous female rat during the delay period. The * indicates a significant difference from the control group $(P<0.05)$. Corticosterone levels increased with the cat and female exposure conditions (middle), but memory errors increased only with the cat-exposure condition (top). Testosterone levels were unaffected by the manipulations (bottom).

arousing experience, independent of its valence (pleasurable or aversive), may affect hippocampal processing.

In this work, rats were fully trained to perform at a high degree of performance in the RAWM to find a hidden platform before any experimental manipulations were conducted. On this baseline of highly accurate baseline performance under control conditions, we found that acute stress (cat exposure) selectively impaired spatial working (hippocampal-dependent) memory, but had no detectable effect on motivation or attentional variables that might influence water-maze performance, as indicated by a lack of effect of cat exposure on long-term (hippocampalindependent) reference memory. In contrast, exposing male rats to a novel environment or to a receptive female rat had no effect on their spatial working memory. Whereas cat and female rat exposure produced an equivalent increase in corticosterone levels, a significant positive correlation between corticosterone levels and memory errors was observed only for the cat-exposed group. Overall, these findings indicate that predator-induced fear, and not merely an increase in arousal, was the critical factor that produced the impairment of hippocampal functioning.

\section{Why Does Cat Exposure Impair Performance by Rats in the Water Maze?}

In previous work, we showed that spatial working memory was impaired in rats exposed to a cat (Diamond et al. 1999b). It was possible that the intense fear induced by cat exposure disrupted the rats' ability to perform the task. The deficits in performance, therefore, could have been produced as a result of nonmnemonic variables, such as impaired attention or increases in general motor activity. The findings of Experiment 1 indicate that the memory impairment seen in rats following cat exposure cannot be explained by a general effect of cat exposure on performance variables, for example, on motor activity or their motivation to escape the water. Whereas rats in both groups had an equivalent number of days of training in the water maze prior to cat exposure, the reference memory-trained rats were unimpaired by cat exposure (Fig. 1). Thus, the stress-induced memory impairment was selective for recently acquired (hippocampal-dependent) spatial working memory. These findings are consistent with previous work from this laboratory showing that stress produced retrograde amnesia by interfering with recently acquired (hippocampal-dependent), but not long-term (hippocampal-independent) spatial memory (Diamond et al. 1996).

One issue left unresolved by this work is whether the stress effects on memory described here were produced by an impairment of the consolidation versus retrieval phases of memory processing (de Quervain et al. 1998; McGaugh and Roozendaal 2002). The consolidation/retrieval distinction could not be addressed here because predator exposure produced an increase in corticosterone levels that remained elevated for the entire duration of the delay period between the learning and acquisition phases. Thus, the impairment of performance on the memory test trial could have occurred because cat exposure and the stressinduced increase in corticosterone levels interfered with storage (i.e., consolidation) and/or retrieval processes. Preliminary findings from our laboratory are relevant to a resolution of this issue. We have increased the delay period between the learning and memory phases of RAWM training from $30 \mathrm{~min}$ to $24 \mathrm{~h}$. In the recent work, we have found that $30 \mathrm{~min}$ of predator exposure, administered immediately after the acquisition phase (on Day 1) had no effect on memory tested $24 \mathrm{~h}$ later (on Day 2). In contrast, predator exposure impaired memory when it was administered for $30 \mathrm{~min}$ before the 24-h memory test (V. Haynes and D. Diamond, unpubl.). Our preliminary findings are consistent with the work of de Quervain et al (1998) who reported that restraint and shock impaired 24-h memory when administered $30 \mathrm{~min}$ before the spatial memory test in water-maze trained rats. The findings are also consistent with views summarized by Roozendaal (2002) that stress or glucocorticoids tend to exert an adverse effect on retrieval and not on consolidation processes. Therefore, our preliminary work with 24 -h memory testing, as with the findings of de Quervain et al (1998) and Roozendaal (2002), indicates that stress exerts a selective adverse effect on retrieval, rather than consolidation processes.

\section{Is It Fear or Arousal That Caused the Spatial Memory Impairment?}

Numerous studies on rodents and people have shown that increased arousal influences the quality and durability of learning and memory (Bradley et al. 1992; Cahill and McGaugh 1995; Diamond et al. 1999b; Blake et al. 2001). Memory modulatory effects of emotion can be produced by stimuli with negative valence, such as fear, as well as by stimuli with positive valence, such as those that evoke a pleasurable response (Christianson 1986; Mazzoni and Loftus 1996; Joslyn et al. 1997; Christianson and Engelberg 1999; Schmidt 2002). Considering the importance of increased arousal alone as a potent modulator of memory, the adverse effects of cat exposure on spatial memory could have been produced by increased arousal, rather than by fear, per se. 


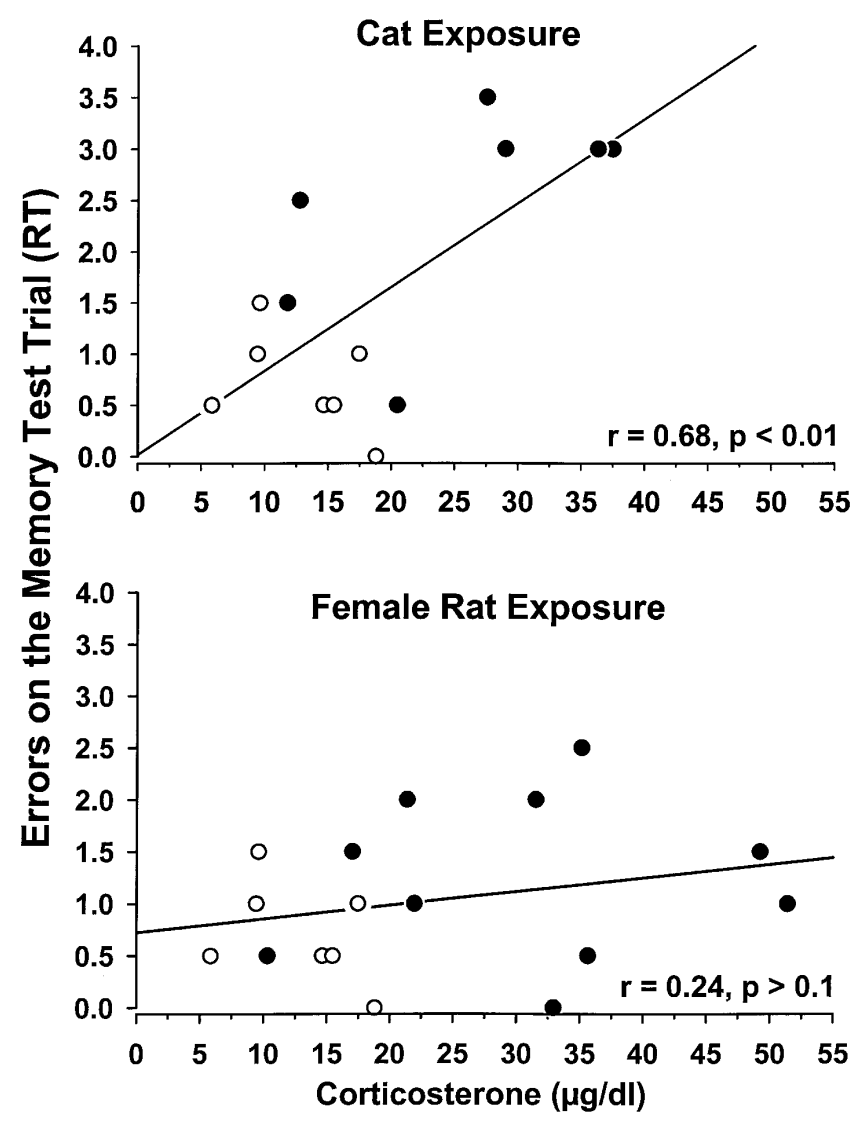

Figure 4 Linear correlations between corticosterone levels and memory errors (RT) for rats exposed to the novel box $(\bigcirc)$ and a cat $(0$; top) or to a female $(\mathbf{0}$; bottom). The top graph illustrates a significant linear relationship between corticosterone and memory for rats in the control + cat exposure condition.

The findings in Experiment 2 do not support the idea that increased arousal alone is sufficient to impair rodent spatial memory. Whereas male rats exposed to the novel environment or to an estrous female during the retention interval were not impaired in their working memory, rats exposed to a cat were impaired. The fact that there was an equivalent increase in corticosterone levels in the female- and cat-exposed groups indicates that both conditions were arousing, that is, they both activated the hypothalamic-pituitary-adrenal axis. Therefore, fear, and not merely increased arousal, appears to be a critical component of the predator-induced impairment of spatial working memory.

The current results may appear to be in conflict with other work showing that exposing an animal to a novel environment alone, impaired spatial memory in food foraging and social recognition tasks (Diamond et al. 1996; Mendl 1999; Burman and Mendl 2000) and blocked the induction of hippocampal synaptic plasticity (Diamond et al. 1990, 1994, 1999a; Xu et al. 1997, 1998). We suggest that differences in the effects of novelty on memory and plasticity arise from differences in the stressfulness of the training tasks. Water-maze training is inherently more stressful than foraging or social recognition tasks (Holscher 1999), potentially rendering water-maze-trained rats less sensitive to mild stressors, such as novelty (see also Dienstbier 1991). Thus, whereas rats trained in relatively low-stress tasks react strongly to novelty, alone, an especially intense stressor, such as cat exposure, may be necessary to impair memory in rats trained in the water maze.

\section{Relation to Previous Studies on Glucocorticoid-Memory Correlations}

Studies on people have found that stress-evoked increases in cortisol have correlated with the degree of impaired memory (Brandenberger et al. 1980; Neylan et al. 2001; Wolf et al. 2001). We present here the novel finding that a comparable process appears to occur in rats; stress-evoked increases in corticosterone correlated with impaired memory in a hippocampal-dependent task. It is relevant that the converse findings of a correlation between enhanced memory in fear conditioning and elevated levels of corticosterone has been reported in rats (Cordero and Sandi 1998; Cordero et al. 1998). Their work, in concert with our findings, indicates that increased corticosterone levels contribute to the enhancement of memories central to the stressful experience (i.e., of the fear-conditioning experience) and to the impairment of memories peripheral to, or outside of the stress context (i.e., the memory of the hidden platform location; Sandi 1998; de Kloet et al. 1999; Diamond et al. 2001; Cordero et al. 2002; Roozendaal 2002).

The correlation described here between corticosterone and impaired memory was not based solely on a concentration-dependent influence of corticosterone on memory. The corticosterone-memory correlation was observed when elevated corticosterone levels were produced in a fear-provoking situation (cat exposure), but not in an appetitive situation (female rat exposure). Comparable findings in other studies have shown that exogenous administration of high doses of corticosterone to otherwise nonstressed rats had no effect on spatial memory in welltrained rats in the RAWM (Park et al. 2001b) or in rats trained in cold $\left(19^{\circ} \mathrm{C}\right)$ water (Sandi et al. 1997).

Our finding that elevated corticosterone levels needed to occur in conjunction with a fear-induced behavioral state to affect memory is relevant to the work of Buchanan and Lovallo (2001) in their study of cortisol-arousal interactions and memory in people. These investigators reported that cortisol administration enhanced memory for emotionally arousing information, but had no effect on memory for emotionally neutral information. Similarly, post-learning stress (cold pressor stress) enhanced memory for arousing information, but had no effect on emotionally neutral information (Cahill et al. 2003). Taken together, these studies suggest that elevated glucocorticoid levels interact with stress-provoking stimuli to modulate the strength of memory formation.

In a related study with people, Brandenberger et al. (1980) also showed a positive correlation between stress-induced increases in cortisol levels and memory. However, whereas Buchanan and Lovallo (2001) showed that elevated levels of cortisol correlated with enhanced memory, Brandenberger et al. (1980) showed that elevated levels of cortisol correlated with impaired memory. The capacity for glucocorticoids to either enhance or impair memory has been a topic of discussion in recent years. Investigators have noted that an increase in glucocorticoid levels appears to contribute to the enhancement of the memory of the stress experience itself, while simultaneously impairing the retrieval of information acquired outside of the stress context (Cordero et al. 1998; Sandi 1998; de Kloet et al. 1999; Diamond et al. 2001; Roozendaal 2002). Thus, high glucocorticoid levels at the time of learning have been shown to enhance the memory of an arousing experience in rats (Roozendaal and McGaugh 1997; Cordero et al. 1998) and people (Buchanan and Lovallo 2001), and to impair memory for information acquired outside of the stress experience (Lupien et al. 1997; Diamond et al. 1999b). Our findings here are consistent with the literature on glucocorticoid-memory interactions in that the information that was forgotten (the platform location) was outside of the stress context (cat exposure). 


\section{Mechanisms Underlying Stress Effects on Memory and Interactions Between Brain Structures}

The current work is relevant toward understanding the mechanisms that underlie stress-induced memory impairments. Previous work has reported correlations between increased levels of corticosterone and a suppression of hippocampal long-term (LTP) and primed burst (PB) potentiation, two electrophysiological models of memory (Diamond et al. 1988; Martin et al. 2000). Foy et al. (1987) first reported that there was a linear correlation between the magnitude of stress-induced increases in corticosterone and the suppression of LTP recorded in vitro, which was then extended by Bennett et al (1991) with recordings in vivo. The work by Foy et al. (1987) and Bennett et al. (1991), was replicated and extended by Diamond et al. (1992) and Kerr et al. (1994), who showed that the overall corticosterone-PB/LTP curve was U-shaped, in which low and high levels of corticosterone correlated with reduced PB and LTP, and intermediate levels of corticosterone correlated with maximal plasticity. Finally, recent work from our group has shown that there is a U-shaped function between corticosterone levels and spatial memory tested in the RAWM (Park et al. 2001b). In the current work, predator exposure produced corticosterone levels at the high end of the Ushaped curve, which may have impaired spatial memory through a suppression of hippocampal synaptic plasticity.

The current findings are also relevant to the observation that corticosterone levels can be elevated under a variety of different conditions, but memory is impaired in only a subset of those conditions. That is, stressful conditions, such as fear-provoking experiences in rats (Diamond et al. 1996, 1999b) and public speaking in people (Kirschbaum et al. 1996; Lupien et al. 1997; Payne et al. 2002), are associated with increases in glucocorticoid levels and with impaired memory. However, elevated corticosterone levels alone are insufficient to block LTP and to impair memory. For example, acute administration of an atypical antidepressant, tianeptine, blocked stress effects on LTP (Shakesby et al. 2002) without reducing the stress-induced rise of corticosterone levels. Moreover, Kim et al. (2001) showed that stress did not block LTP or impair memory in rats with damage to the amygdala, despite the fact that the stressed amygdala-lesioned rats had a normal stress-induced increase in corticosterone levels. Complementary work by Roozendaal (2000) and Roozendall et al. (2003) reported that corticosterone effects on hippocampus-dependent memory could be blocked by inactivation or lesioning of the amygdala. We have also shown that exogenous corticosterone administration to otherwise nonstressed rats did not impair spatial memory (Park et al. 2001b). Our current findings provide a clear example of how elevated corticosterone levels alone do not control hippocampal functioning. The concentration-dependent correlation between impaired memory and corticosterone reported here was expressed only when elevated levels of corticosterone occurred during a fear-induced behavioral state produced by predator exposure.

This view of a permissive influence of stress on the expression of glucocorticoid effects on memory is relevant to the finding that an acute elevation of glucocorticoid levels can occur under a variety of different conditions, for example, such as during feeding, exercise, and sexual activity (Moberg et al. 1975; Phoenix et al. 1977; Bronson and Desjardins 1982; Rosmond et al. 2000; Kanaley et al. 2001; Makatsori et al. 2003), but such activities don't typically produce cognitive impairments. By extension, our work predicts that PB and LTP would not be suppressed under conditions in which elevated glucocorticoid levels would be produced under nonstress conditions, for example, by exogenous corticosterone administration to nonstressed rats or by appetitive experiences, for example, female exposure or feeding.
The permissive influence of fear in the expression of the corticosterone-memory correlation is likely to involve an influence of the amygdala on the hippocampus. Amygdala activation is necessary for the modulation of memory by glucocorticoids (Roozendaal and McGaugh 1997; Kim et al. 2001; Roozendaal et al. 2001), as well as for the stress-induced suppression of hippocampal LTP (Akirav and Richter-Levin 1999; Richter-Levin and Akirav 2000; Kim et al. 2001). It is therefore likely that the circuitry involved in predator stress effects on memory includes corticosteroid, as well as noradrenergic, activation of the amygdala (McGaugh and Roozendaal 2002; Roozendaal 2002), in concert with local inhibitory effects of corticosterone on the hippocampus (Pavlides et al. 1996; Pavlides and McEwen 1999; Joels 2001).

Finally, it is important to note that fear and appetitive behaviors (e.g., sexual activity and self-administration of drugs), can activate the same brain structures, such as the amygdala, nucleus accumbens and hypothalamus (Veening and Coolen 1998; Hamann et al. 1999, 2002; Dielenberg and McGregor 2001; Heeb and Yahr 2001; Levita et al. 2002; Kippin et al. 2003; Singewald et al. 2003), but here, only the fear-provoking stimulus (predator exposure) impaired memory. A complete understanding of why a fear-provoking, but not appetitive, stimulus-impaired memory will need to take into account the activation of different subnuclei within emotional brain circuitry, as well as different neurochemical manifestations of fear-provoking, versus appetitive, experiences (e.g., Comoli et al. 2003).

\section{Summary}

This series of experiments was designed to extend our understanding of how emotionality affects memory-related functioning of the hippocampus. We found that rats trained in a hippocampal-dependent (working) memory task, but not rats trained in a hippocampal-independent (reference) memory task, were impaired by acute stress. We also demonstrated that the memoryimpairing effects of arousal were selective to a fear-provoking situation (cat exposure), and not to an arousing situation that lacked a fear component (estrous female rat exposure). Finally, we found that there was a significant correlation between memory deficits and corticosterone levels in the predator stress condition, but not in the female rat exposure condition. Overall, these findings provide support for the idea that activation of fear circuits of the brain interacts with elevated levels of corticosterone to interfere with hippocampal-dependent memory.

\section{MATERIALS AND METHODS}

\section{Animals, Radial-Arm Water Maze, and Working-Memory Training}

Adult male Harlan Sprague-Dawley rats (250-275g) were given 1 wk to acclimate to the vivarium 12:12 light/dark cycle (lights off at $6 \mathrm{AM}$ ) with food and water available ad libitum. Methodological procedures involved in training rats in the radial-arm water maze (RAWM) have been described previously (Diamond et al. 1999b; Park et al. 2001a). In brief, training took place in a black tank $(168 \mathrm{~cm}$ diameter, height $56 \mathrm{~cm}$ ) filled with clear water $\left(21 \pm 2^{\circ} \mathrm{C}\right)$ with internal walls positioned to produce six swim paths or arms radiating out of an open central area (for review, see Diamond et al. 1999b; Arendash et al. 2001; Park et al. 2001a, for illustrations of the RAWM). The arm that contained the hidden escape platform, located $2 \mathrm{~cm}$ below the surface of the water, is referred to as the goal arm. Working-memory training in the RAWM is similar in concept to the strategy used in previous studies utilizing the open-field (Morris) water maze (Whishaw 1985; Bohbot et al. 1996; Steele and Morris 1999) in that rats were trained to learn a new location of the hidden platform each 
day. In the RAWM, the number of incorrect arm entries on each trial served as the operational measure of learning and memory. On each day of training, the rats were transported from the vivarium into the laboratory, and 45 min later, water-maze training began. At the start of each training trial, a rat was placed in the water in an arm that didn't contain the platform (start arm) at the entrance to the central area of the maze. The rat swam out of the start arm into the open central area, and then it would swim into any of the six arms of the maze. Entries into arms other than the goal arm were errors. Rats were given up to $1 \mathrm{~min}$ per trial to find the platform, and then they were allowed to remain on it for 20 sec. Rats that didn't find the platform in the 1 -min period were guided gently to the platform by the experimenter. The goal arm was different across rats within a day to eliminate a scent buildup in the vicinity of the hidden platform. All testing took place during the dark cycle (between 9 AM and 4 PM).

Each day, the rats were given a total of eight training trials (T1-T8) in two groups of four trials. Between the two groups of four trials, was a delay period that lasted for 30 (Experiment 1 ) or 45 (Experiment 2) min. The first four trials (T1-T4) gave the rats the opportunity to learn where the platform was located on that day (Acquisition Phase). The duration of T1-T4 in well-trained rats was $\sim 2-3 \mathrm{~min}$. After T4, the rats were removed from the maze and placed either in their home cages (control condition), or they were given an experimental manipulation (described below and in the specific methods for each experiment).

During preliminary daily training in all experiments, rats spent the delay period in their home cages. At the end of the delay period, the rats were returned to the RAWM and were given Trials 5-8 (Retention Phase; T5-T8). The first trial in the retention phase (T5) was the critical memory test trial, because good performance on this trial could occur only if the rats had learned (on T1-T4), and then remembered across the delay period, the location of the hidden platform. To illustrate the importance of T5 as the memory test trial, throughout the text and in Figs. 1-4, T5 is referred to as the retention trial (RT). In some instances, performance on the RT was impaired by the experimental manipulation performed during the delay period. The purpose of the three additional trials (T6-T8) was to detect whether the rats could relearn the location of the hidden platform.

In summary, under working-memory training conditions, the escape platform was always located at the end of the same arm on each of the eight trials within a day. Across days, the platform was pseudo-randomly located in any one of the six arms, with the exception that the platform was never located in the same arm on two consecutive days. The training procedures, therefore, tested hippocampal-dependent (working) memory because the rats had to learn (on T1-T4), and then remember (tested on the RT after the 30- or 45-min delay period), which arm contained the hidden platform on that day.

\section{Stress Manipulation}

As described previously, cat exposure served as the stressor, because it produces an instinctual fear response in rats (Blanchard et al. 1993). Rats were placed in a ventilated Plexiglas rat pie-a round chamber $(42 \mathrm{~cm}$ diameter, $6.75 \mathrm{~cm}$ height; Braintree Scientific) comprised of 12 wedge-shaped compartments, to expose them to the visual, auditory, and olfactory components of the adult female cat, or female rat (frustration group), while eliminating any possibility of physical contact between the animals. Each wedge $(21 \times 21 \times 11 \mathrm{~cm})$ had sufficient room for one rat to move freely within its compartment. The pie-chamber with the rats and the cat were all contained in a large wooden box $(75 \times 57 \times 58 \mathrm{~cm})$, which had a see-through door, an exhaust fan, and a 60-watt house light in the ceiling. The cat was fed moist fish-flavored cat food while it was in the chamber, which helped to keep it active throughout the exposure period. In Experiment 2 , cat-exposed rats were placed in the Plexiglas rat-pie container, and female rat-exposed subjects were placed within a ventilated Plexiglas box $(28 \times 9 \times 14 \mathrm{~cm})$. Rats in the Novel Box group were placed alone in the same chamber that was used for female rat exposure.

\section{Performance Criterion}

The experimental manipulations were conducted only on rats that first performed at a high degree of accuracy under control conditions, as we have described previously (Diamond et al. 1999b). To meet the performance criterion, a rat could commit a total of no more than one error on the first retention test trial (RT) across three consecutive days of training. That is, a rat could score two zeros and one error in any order, or have three consecutive days with zero errors on the RT to meet the performance criterion. The day in which a rat satisfied the 3-d criterion period is referred to as its "day to criterion" (DTC). As we have discussed in previous work, chance level of performance in a 6-arm maze is 2.5 errors/trial for an animal that does not make perseverative errors, that is, for an animal that makes only one entry per arm until it finds the platform, as is found in hippocampal-lesioned rats (Diamond et al. 1999b). During 3 d of testing, an animal performing at chance without making perseverative errors would be expected to commit 2.5 errors/day, for a total of 7.5 errors in a 3-d period. Thus, for a rat to commit a total of no more than one error on the RT over $3 \mathrm{~d}$ of training, demanded a high degree of within-day spatial learning and memory capacity. This stringent performance criterion ensured that the experimental manipulations were administered only to rats that had exhibited excellent spatial working memory on three consecutive days under control conditions.

\section{Statistics}

All experimental manipulations were carried out during the 30(Experiment 1) or 45-min (Experiment 2) delay period on the two days after each rat reached its DTC, and the same manipulation was conducted on each rat on both test days. As we have found previously (Diamond et al. 1999b), there were no significant differences in performance on the first versus the second day of post-DTC testing. Therefore, the errors committed by the rats on the two post-DTC test days were combined to form a single mean value for each rat.

Rats that did not reach the performance criterion were not included in this study. Outlier data were excluded if they were more than five standard deviations from the exclusive mean (less than $1 \%$ of the data were outliers). Mixed design-repeated measures ANOVAs were conducted on the acquisition phase (T1-T4) and retention phase (RT-T8), followed by Bonferroni-corrected $t$-tests. Significance level was $P<0.05$. Because our prior work indicated that stress produces an impairment of performance on the RT (Diamond et al. 1996, 1999b), we conducted planned pairwise comparisons on the RT. In addition, Trials 6-8 were important because they provided a measure of relearning after an impairment occurred on the RT. Therefore, planned comparisons between relevant groups on T6-T8 were performed whenever there was a significant impairment of performance on the RT.

\section{Experiment 1 Methods}

The procedures used in this experiment were the same as described in the above, with the exception that there were three groups of rats, two received WM training and one received RM training. One of the WM-trained groups was exposed to the cat (WM-Cat; $n=9$ ), and the other WM-trained group was placed in the home cage (WM-HC; $n=7$ ) during the 30 -min delay period between T4 and the RT on the two post-DTC test days. The RM trained group (RM-Cat; $n=8$ ) went to a constant platform location for the same mean number of days of preliminary training that the two WM groups received before their post-DTC testing began $(12 \mathrm{~d})$. This design equalized the number of days of preliminary training all groups received before the experimental manipulation, that is, cat exposure, was given during the 30-min delay period. Thus, for WM-trained rats, the goal arm was the same on every trial within a day and was different across days. For RM-trained rats, the goal arm was at the same location on every trial for every one of the twelve days of preliminary training and on the subsequent two days of stress-memory testing. For all animals in all groups, the start arm was different on every trial 
within a day to force the rats to use a spatial, rather than a motor or response, strategy to locate the hidden platform.

\section{Experiment 2 Methods}

As in both experiments, the subjects were virgin adult-male rats with no post-weaning exposure to females prior to RAWM testing. All animals were trained using standard WM-testing procedures (described above), except that the delay period in Experiment 2 was 45 min. During preliminary daily training, all rats spent the 45-min delay period (between T4 and T5) in their home cages. On each of the two test days after the rats reached their DTC, they spent the first 30 min of the delay period in an experimental condition (with a cat, female rat, or in a novel environment), after which all rats spent the final $15 \mathrm{~min}$ of the delay period in their home cages. The delayed return to the water maze was implemented in this experiment in an attempt to potentially dissociate effects of heightened emotionality from impaired memory, per se. It was possible that a post-cat 15-min cool down period by the rats in their home cages might help them to recover from the cat exposure experience, thereby enabling the cat-exposed rats to exhibit normal memory in the RAWM.

Eight female Sprague-Dawley rats were used solely as sexual stimuli. The females were ovariectomized and then primed with an ovarian hormone regimen known to reliably induce a state of behavioral estrus (Woodson and Balleine 2002). The timing of the induced estrus coincided with the two post-DTC days of testing for each of the males in the female-exposed group. All females were prescreened with a nonexperimental male before their use as stimulus animals, and females exhibiting a lordosis quotient of $<100 \%$ (10 lordosis responses to 10 complete mounts by a male) were not used that day. Exposure of each male to an estrous female took place in a clear Plexiglas-walled, $46-\mathrm{cm}$ square observation chamber with bedding. Interactions between males and females were videotaped and then stereotypic characteristics of male copulatory behaviors were quantified as described elsewhere (Woodson and Balleine 2002). Exposure of the males to the females was conducted either with $(n=5)$ or without $(n=5)$ physical contact allowed. The latter group was placed in a ventilated Plexiglas isolation box $(28 \times 14 \times 9 \mathrm{~cm})$ inside the larger chamber containing the female.

Rats in the other two groups were also placed individually in the same Plexiglas isolation box, except that they were put either in a large chamber with a cat $(n=7)$ or in a novel location within the maze training room $(n=7)$.

\section{Blood Sampling}

Tail blood samples $(0.5 \mathrm{~mL})$ were obtained immediately after the memory test trial on the second day of post-DTC testing. After clotting and centrifugation, the serum was extracted and stored at $-70^{\circ} \mathrm{C}$ until it was assayed for corticosterone and testosterone by radioimmunoassay (ICN pharmaceuticals).

\section{ACKNOWLEDGMENTS}

This research was supported by a Merit Review Grant from the Department of Veterans' Affairs to D.M.D.

The publication costs of this article were defrayed in part by payment of page charges. This article must therefore be hereby marked "advertisement" in accordance with 18 USC section 1734 solely to indicate this fact.

\section{REFERENCES}

Akirav, I. and Richter-Levin, G. 1999. Biphasic modulation of hippocampal plasticity by behavioral stress and basolateral amygdala stimulation in the rat. J. Neurosci. 19: 10530-10535.

Alfarez, D.N., Wiegert, O., Joels, M., and Krugers, H.J. 2002. Corticosterone and stress reduce synaptic potentiation in mouse hippocampal slices with mild stimulation. Neuroscience 115: 1119-1126.

Arendash, G.W., King, D.L., Gordon, M.N., Morgan, D., Hatcher, J.M., Hope, C.E., and Diamond, D.M. 2001. Progressive, age-related behavioral impairments in transgenic mice carrying both mutant amyloid precursor protein and presenilin-1 transgenes. Brain Res. 891: $42-53$.

Bennett, M.C., Diamond, D.M., Fleshner, M., and Rose, G.M. 1991. Serum corticosterone level predicts the magnitude of hippocampal primed burst potentiation and depression in urethane-anesthetized rats. Psychobiology 19: 301-307.

Bindra, D. 1959. Stimulus change, reactions to novelty, and response decrement. Psychol. Rev. 66: 96-103.

Blake, T.M., Varnhagen, C.K., and Parent, M.B. 2001. Emotionally arousing pictures increase blood glucose levels and enhance recall. Neurobiol. Learn. Mem. 75: 262-273.

Blanchard, R.J., Yudko, E.B., Rodgers, R.J., and Blanchard, D.C. 1993. Defense system psychopharmacology: An ethological approach to the pharmacology of fear and anxiety. Behav. Brain Res. 58: 155-165.

Bohbot, V., Otahal, P., Liu, Z., Nadel, L., and Bures, J. 1996. Electroconvulsive shock and lidocaine reveal rapid consolidation of spatial working memory in the water maze. Proc. Natl. Acad. Sci. 93: 4016-4019.

Bradley, M.M., Greenwald, M.K., Petry, M.C., and Lang, P.J. 1992. Remembering pictures-pleasure and arousal in memory. J. Exper. Psychol. Learn. Mem. Cogn. 18: 379-390.

Brandenberger, G., Follenius, M., Wittersheim, G., and Salame, P. 1980. Plasma catecholamines and pituitary adrenal hormones related to mental task demand under quiet and noise conditions. Biol. Psychol. 10: $239-252$.

Bronson, F.H. and Desjardins, C. 1982. Endocrine responses to sexual arousal in male mice. Endocrinology 111: 1286-1291.

Buchanan, T.W. and Lovallo, W.R. 2001. Enhanced memory for emotional material following stress-level cortisol treatment in humans. Psychoneuroendocrinology 26: 307-317.

Burman, O.H.P. and Mendl, M. 2000. Short-term social memory in the laboratory rat: Its susceptibility to disturbance. Appl. Anim. Behav. Sci. 67: 241-254.

Bush, S.I. and Geer, J.H. 2001. Implicit and explicit memory of neutral, negative emotional, and sexual information. Arch. Sex. Behav. 30: $615-631$.

Cahill, L. 2000. Neurobiological mechanisms of emotionally influenced, long-term memory. Cognition, Emotion and Autonomic Responses: The Integrative Role of the Prefrontal Cortex and Limbic Structures. Prog. Brain Res. 126: 29-37.

Cahill, L. and McGaugh, J.L. 1995. A novel demonstration of enhanced memory associated with emotional arousal. Conscious. Cogn. 4: $410-421$.

Cahill, L., Gorski, L., and Le, K. 2003. Enhanced human memory consolidation with post-learning stress: Interaction with the degree of arousal at encoding. Learn. Mem. 10: 270-274.

Christianson, S.A. 1986. Effects of positive emotional events on memory. Scandinavian J. Psychol. 27: 287-299.

Christianson, S.A. and Engelberg, E. 1999. Memory and emotional consistency: The MS Estonia ferry disaster. Memory 7: 471-482.

Comoli, E., Ribeiro-Barbosa, E.R., and Canteras, N.S. 2003. Predatory hunting and exposure to a live predator induce opposite patterns of Fos immunoreactivity in the PAG. Behav. Brain Res. 138: 17-28.

Cordero, M.I. and Sandi, C. 1998. A role for brain glucocorticoid receptors in contextual fear conditioning: Dependence upon training intensity. Brain Res. 786: 11-17.

Cordero, M.I., Merino, J.J., and Sandi, C. 1998. Correlational relationship between shock intensity and corticosterone secretion on the establishment and subsequent expression of contextual fear conditioning. Behav. Neurosci. 112: 885-891.

Cordero, M.I., Kruyt, N.D., Merino, J.J., and Sandi, C. 2002. Glucocorticoid involvement in memory formation in a rat model for traumatic memory. Stress 5: 73-79.

de Kloet, E.R., Oitzl, M.S., and Joels, M. 1999. Stress and cognition: Are corticosteroids good or bad guys? Trends Neurosci. 22: 422-426.

de Quervain, D.J., Roozendaal, B., and McGaugh, J.L. 1998. Stress and glucocorticoids impair retrieval of long-term spatial memory. Nature 394: 787-790.

de Quervain, D.J., Roozendaal, B., Nitsch, R.M., McGaugh, J.L., and Hock, C. 2000. Acute cortisone administration impairs retrieval of long-term declarative memory in humans. Nat. Neurosci. 3: 313-314.

Diamond, D.M. and Park, C.R. 2000. Predator exposure produces retrograde amnesia and blocks synaptic plasticity. Progress toward understanding how the hippocampus is affected by stress. Ann. NY Acad. Sci. 911: 453-455.

Diamond, D.M., Dunwiddie, T.V., and Rose, G.M. 1988. Characteristics of hippocampal primed burst potentiation in vitro and in the awake rat. J. Neurosci. 8: 4079-4088.

Diamond, D.M., Bennett, M.C., Stevens, K.E., Wilson, R.L., and Rose, G.M. 1990. Exposure to a novel environment interferes with the induction of hippocampal primed burst potentiation in the behaving rat. Psychobiology 18: 273-281.

\section{Learning \& Memory}


Diamond, D.M., Bennett, M.C., Fleshner, M., and Rose, G.M. 1992. Inverted-U relationship between the level of peripheral corticosterone and the magnitude of hippocampal primed burst potentiation. Hippocampus 2: 421-430.

Diamond, D.M., Fleshner, M., and Rose, G.M. 1994. Psychological stress repeatedly blocks hippocampal primed burst potentiation in behaving rats. Behav. Brain Res. 62: 1-9.

Diamond, D.M., Fleshner, M., Ingersoll, N., and Rose, G.M. 1996. Psychological stress impairs spatial working memory: Relevance to electrophysiological studies of hippocampal function. Behav. Neurosci. 110: 661-672.

Diamond, D.M., Fleshner, M., and Rose, G.M. 1999a. The enhancement of hippocampal primed burst potentiation by dehydroepiandrosterone sulfate (DHEAS) is blocked by psychological stress. Stress 3: 107-121.

Diamond, D.M., Park, C.R., Heman, K.L., and Rose, G.M. 1999b. Exposing rats to a predator impairs spatial working memory in the radial arm water maze. Hippocampus 9: 542-552.

Diamond, D.M., Park, C.R., Puls, M.J., and Rose, G.M. 2001. Differential effects of stress on hippocampal and amygdaloid LTP: Insight into the neurobiology of traumatic memories. In: Neuronal mechanisms of memory formation (ed. C. Holscher), pp 379-403. Cambridge University Press, New York.

Dielenberg, R.A. and McGregor, I.S. 2001. Defensive behavior in rats towards predatory odors: A review. Neurosci. Biobehav. Rev. 25: 597-609.

Dielenberg, R.A., Carrive, P., and McGregor, I.S. 2001. The cardiovascular and behavioral response to cat odor in rats: Unconditioned and conditioned effects. Brain Res. 897: 228-237.

Dienstbier, R.A. 1991. Behavioral correlates of sympathoadrenal reactivity: The toughness model. Med. Sc. Sports Exer. 23: 846-852.

Eichenbaum, H., Stewart, C., and Morris, R.G. 1990. Hippocampal representation in place learning. J. Neurosci. 10: 3531-3542.

Foy, M.R., Stanton, M.E., Levine, S., and Thompson, R.F. 1987. Behavioral stress impairs long-term potentiation in rodent hippocampus. Behav. Neural. Biol. 48: 138-149.

Hamann, S.B., Ely, T.D., Grafton, S.T., and Kilts, C.D. 1999. Amygdala activity related to enhanced memory for pleasant and aversive stimuli. Nat. Neurosci. 2: 289-293.

Hamann, S.B., Ely, T.D., Hoffman, J.M., and Kilts, C.D. 2002. Ecstasy and agony: Activation of the human amygdala in positive and negative emotion. Psychol. Sci. 13: 135-141.

Heeb, M.M. and Yahr, P. 2001. Anatomical and functional connections among cell groups in the gerbil brain that are activated with ejaculation. J. Comp. Neurol. 439: 248-258.

Holscher, C. 1999. Stress impairs performance in spatial water maze learning tasks. Behav. Brain Res. 100: 225-235.

Joels, M. 2001. Corticosteroid actions in the hippocampus. J. Neuroendocrinol. 13: 657-669.

Joseph, R. 1999. The neurology of traumatic "dissociative" amnesia: Commentary and literature review. Child Abuse \& Neglect 23: $715-727$.

Joslyn, S., Carlin, L., and Loftus, E.F. 1997. Remembering and forgetting childhood sexual abuse. Memory 5: 703-724.

Kanaley, J.A., Weltman, J.Y., Pieper, K.S., Weltman, A., and Hartman, M.L. 2001. Cortisol and growth hormone responses to exercise at different times of day. J. Clin. Endocrinol. Metab. 86: 2881-2889.

Kerr, D.S., Huggett, A.M., and Abraham, W.C. 1994. Modulation of hippocampal long-term potentiation and long-term depression by corticosteroid receptor activation. Psychobiology 22: 123-133.

Kim, J.J. and Diamond, D.M. 2002. The stressed hippocampus, synaptic plasticity and lost memories. Nat. Rev. Neurosci. 3: 453-462.

Kim, J.J., Lee, H.J., Han, J.S., and Packard, M.G. 2001. Amygdala is critical for stress-induced modulation of hippocampal long- term potentiation and learning. J. Neurosci. 21: 5222-5228.

Kippin, T.E., Cain, S.W., and Pfaus, J.G. 2003. Estrous odors and sexually conditioned neutral odors activate separate neural pathways in the male rat. Neuroscience 117: 971-979.

Kirschbaum, C., Wolf, O.T., May, M., Wippich, W., and Hellhammer, D.H. 1996. Stress- and treatment-induced elevations of cortisol levels associated with impaired declarative memory in healthy adults. Life Sci. 58: $1475-1483$.

Kitajima, I., Yamamoto, T., Ohno, M., and Ueki, S. 1992. Working and reference memory in rats in the three-panel runway task following dorsal hippocampal lesions. Jpn. J. Pharmacol. 58: 175-183.

Lane, R.D., Chua, P.M., and Dolan, R.J. 1999. Common effects of emotional valence, arousal and attention on neural activation during visual processing of pictures. Neuropsychologia 37: 989-997.

LeDoux, J.E. 2000. Emotion circuits in the brain. Annu. Rev. Neurosci. 23: $155-184$.

Levita, L., Dalley, J.W., and Robbins, T.W. 2002. Nucleus accumbens dopamine and learned fear revisited: A review and some new findings. Behav. Brain Res. 137: 115-127.

Loftus, E.F. and Kaufman, L. 1992. Why do traumatic experiences sometimes produce good memory (flashbulbs) and sometimes no memory (repression). In: Affect and accuracy in recall (eds. E. Winograd and U. Neisser), pp. 212-226. Cambridge University Press, New York.

Lupien, S.J. and Lepage, M. 2001. Stress, memory, and the hippocampus: Can't live with it, can't live without it. Behav. Brain Res. 127: 137-158.

Lupien, S.J. and McEwen, B.S. 1997. The acute effects of corticosteroids on cognition: Integration of animal and human model studies. Brain Res. Rev. 24: 1-27.

Lupien, S.J., Gaudreau, S., Tchiteya, B.M., Maheu, F., Sharma, S., Nair, N.P., Hauger, R.L., McEwen, B.S., and Meaney, M.J. 1997. Stress-induced declarative memory impairment in healthy elderly subjects: Relationship to cortisol reactivity. J. Clin. Endocrinol. Metab. 82: 2070-2075.

Makatsori, A., Duncko, R., Schwendt, M., Moncek, F., Johansson, B.B., and Jezova, D. 2003. Voluntary wheel running modulates glutamate receptor subunit gene expression and stress hormone release in Lewis rats. Psychoneuroendocrinology 28: 702-714.

Martin, S.J., Grimwood, P.D., and Morris, R.G. 2000. Synaptic plasticity and memory: An evaluation of the hypothesis. Annu. Rev. Neurosci. 23: 649-711.

Mazzoni, G.A. and Loftus, E.F. 1996. When dreams become reality. Conscious Cogn. 5: 442-462.

McGaugh, J.L. 2000. Memory-A century of consolidation. Science 287: $248-251$.

McGaugh, J.L. and Roozendaal, B. 2002. Role of adrenal stress hormones in forming lasting memories in the brain. Curr. Opin. Neurobiol. 12: $205-210$.

Mendl, M. 1999. Performing under pressure: Stress and cognitive function. App. Anim. Behav. Sci. 65: 221-244.

Mesches, M.H., Fleshner, M., Heman, K.L., Rose, G.M., and Diamond, D.M. 1999. Exposing rats to a predator blocks primed burst potentiation in the hippocampus in vitro. J. Neurosci. 19: RC18.

Miller, D.B. and O'Callaghan, J.P. 2002. Neuroendocrine aspects of the response to stress. Metabolism 51: 5-10.

Moberg, G.P., Bellinger, L.L., and Mendel, V.E. 1975. Effect of meal feeding on daily rhythms of plasma corticosterone and growth hormone in the rat. Neuroendocrinology 19: 160-169.

Newcomer, J.W., Selke, G., Melson, A.K., Hershey, T., Craft, S., Richards, K., and Alderson, A.L. 1999. Decreased memory performance in healthy humans induced by stress-level cortisol treatment. Arch. Gen. Psychiatry 56: 527-533.

Newell, S.J., Henderson, K.V., and Wu, B.T. 2001. The effects of pleasure and arousal on recall of advertisements during the Super Bowl. Psychol. Market. 18: 1135-1153.

Neylan, T.C., Canick, J.D., Hall, S.E., Reus, V.I., Sapolsky, R.M., and Wolkowitz, O.M. 2001. Cortisol levels predict cognitive impairment induced by electroconvulsive therapy. Biol. Psychiatry 50: 331-336.

Niikura, S., Yokoyama, O., Komatsu, K., Yotsuyanagi, S., Mizuno, T., and Namiki, M. 2002. A causative factor of copulatory disorder in rats following social stress. J. Urol. 168: 843-849.

Ohno, M., Yamamoto, T., and Watanabe, S. 1993. Blockade of hippocampal nicotinic receptors impairs working memory but not reference memory in rats. Pharmacol. Biochem. Behav. 45: 89-93.

Olton, D.S. and Papas, B.C. 1979. Spatial memory and hippocampal function. Neuropsychologia 17: 669-682.

Olton, D.S., Collison, C., and Werz, M.A. 1977. Spatial memory and radial arm maze performance of rats. Learn. Motiv. 8: 289-314.

Olton, D.S., Becker, J.T., and Handelmann, G.E. 1979. Hippocampus, space, and memory. Behav. Brain Sci. 2: 313-365.

Park, C.R., Campbell, A.M., and Diamond, D.M. 2001a. Chronic psychosocial stress impairs learning and memory and increases sensitivity to yohimbine in adult rats. Biol. Psychiatry 50: 994-1004.

Park, C.R., Campbell, A.M., Fleshner, M., Smith, T.P., Wilbanks, A.L., and Diamond, D.M. 2001b. U-shaped function between corticosterone and spatial memory in stressed rats. FASEB J. 15: A66.

Pavlides, C. and McEwen, B.S. 1999. Effects of mineralocorticoid and glucocorticoid receptors on long-term potentiation in the CA3 hippocampal field. Brain Res. 851: 204-214.

Pavlides, C., Ogawa, S., Kimura, A., and McEwen, B.S. 1996. Role of adrenal steroid mineralocorticoid and glucocorticoid receptors in long-term potentiation in the CA1 field of hippocampal slices. Brain Res. 738: 229-235.

Payne, J.D., Nadel, L., Allen, J.J., Thomas, K.G., and Jacobs, W.J. 2002. The effects of experimentally induced stress on false recognition. Memory 10: $1-6$.

Phoenix, C.H., Dixson, A.F., and Resko, J.A. 1977. Effects of ejaculation on levels of testosterone, cortisol, and luteinizing hormone in peripheral plasma of rhesus monkeys. J. Comp. Physiol. Psychol. 
91: $120-127$

Richardson, R., Siegel, M.A., and Campbell, B.A. 1988. Unfamiliar environments impair information processing as measured by behavioral and cardiac orienting responses to auditory stimuli in preweanling and adult rats. Dev. Psychobiol. 21: 491-503.

Richter-Levin, G. and Akirav, I. 2000. Amygdala-hippocampus dynamic interaction in relation to memory. Mol. Neurobiol. 22: 11-20.

Roozendaal, B. 2000. Glucocorticoids and the regulation of memory consolidation. Psychoneuroendocrinology 25: 213-238.

. 2002. Stress and memory: Opposing effects of glucocorticoids on memory consolidation and memory retrieval. Neurobiol. Learn. Mem. 78: $578-595$.

Roozendaal, B. and McGaugh, J.L. 1997. Basolateral amygdala lesions block the memory-enhancing effect of glucocorticoid administration in the dorsal hippocampus of rats. Euro. J. Neurosci. 9: 76-83.

Roozendaal, B., de Quervain, D.J., Ferry, B., Setlow, B., and McGaugh, J.L. 2001. Basolateral amygdala-nucleus accumbens interactions in mediating glucocorticoid enhancement of memory consolidation. $J$. Neurosci. 21: 2518-2525.

Roozendaal, B., Griffith, Q.K., Buranday, J., de Quervain, D.J., and McGaugh, J.L. 2003. The hippocampus mediates glucocorticoid-induced impairment of spatial memory retrieval: Dependence on the basolateral amygdala. Proc. Natl. Acad. Sci. 100: $1328-1333$.

Rosmond, R., Holm, G., and Bjorntorp, P. 2000. Food-induced cortisol secretion in relation to anthropometric, metabolic and haemodynamic variables in men. Int. J. Obes. Relat. Metab. Disord. 24: $416-422$.

Sandi, C. 1998. The role and mechanisms of action of glucocorticoid involvement in memory storage. Neural Plast. 6: 41-52.

Sandi, C., Loscertales, M., and Guaza, C. 1997. Experience-dependent facilitating effect of corticosterone on spatial memory formation in the water maze. Euro. J. Neurosci. 9: 637-642.

Schmidt, S.R. 2002. Outstanding memories: The positive and negative effects of nudes on memory. J. Exp. Psychol. Learn. Mem. Cogn. 28: $353-361$.
Shakesby, A.C., Anwyl, R., and Rowan, M.J. 2002. Overcoming the effects of stress on synaptic plasticity in the intact hippocampus: Rapid actions of serotonergic and antidepressant agents. J. Neurosci. 22: $3638-3644$

Singewald, N., Salchner, P., and Sharp, T. 2003. Induction of c-Fos expression in specific areas of the fear circuitry in rat forebrain by anxiogenic drugs. Biol. Psychiatry 53: 275-283.

Sokolov, N. 1963. Perception and the conditioned reflex. Pergamon Press, New York.

Steele, R.J. and Morris, R.G. 1999. Delay-dependent impairment of a matching-to-place task with chronic and intrahippocampal infusion of the NMDA-antagonist D-AP5. Hippocampus 9: 118-136.

Veening, J.G. and Coolen, L.M. 1998. Neural activation following sexual behavior in the male and female rat brain. Behav. Brain Res. 92: 181-193.

Whishaw, I.Q. 1985. Formation of a place learning-set by the rat: A new paradigm for neurobehavioral studies. Physiol. Behav. 35: 139-143.

Wolf, O.T., Schommer, N.C., Hellhammer, D.H., McEwen, B.S., and Kirschbaum, C. 2001. The relationship between stress induced cortisol levels and memory differs between men and women. Psychoneuroendocrinology 26: 711-720.

Woodson, J.C. and Balleine, B.W. 2002. An assessment of factors contributing to instrumental performance for sexual reward in the rat. Q. J. Exp. Psychol. B 55: 75-88.

Xu, L., Anwyl, R., and Rowan, M.J. 1997. Behavioural stress facilitates the induction of long-term depression in the hippocampus. Nature 387: 497-500.

Xu, L., Holscher, C., Anwyl, R., and Rowan, M.J. 1998. Glucocorticoid receptor and protein/RNA synthesis-dependent mechanisms underlie the control of synaptic plasticity by stress. Proc. Natl. Acad. Sci. 95: $3204-3208$.

Received May 2, 2003; accepted in revised form July 29, 2003.

\section{Learning \& Memory}



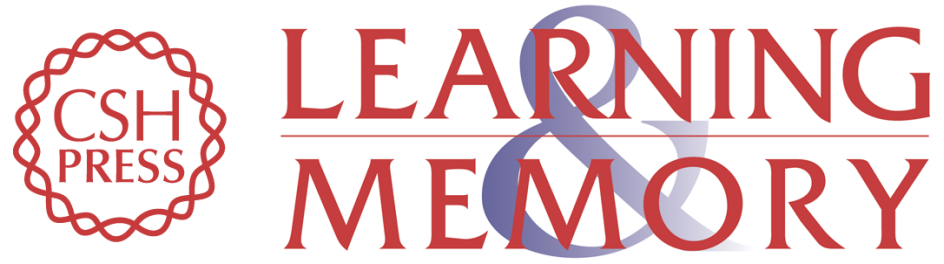

\section{Emotion-Induced Amnesia in Rats: Working Memory-Specific Impairment, Corticosterone-Memory Correlation, and Fear Versus Arousal Effects on Memory}

James C. Woodson, Deric Macintosh, Monika Fleshner, et al.

Learn. Mem. 2003, 10:

Access the most recent version at doi:10.1101/lm.62903

References This article cites 100 articles, 11 of which can be accessed free at:

http://learnmem.cshlp.org/content/10/5/326.full.html\#ref-list-1

License

Email Alerting

Receive free email alerts when new articles cite this article - sign up in the box at the Service top right corner of the article or click here. 